\title{
Ganz gewöhnliche Viertel
}

\author{
Stigma und Realitäten in Casablancas Slum Er-Rhamna
}

\author{
Raffael Beier
}

Slums gelten als das Symbol der vermeintlich unterentwickelten und unkontrolliert wachsenden Megastädte des Globalen Südens. Die damit einhergehende Stigmatisierung von Slums hat nicht nur alltägliche Folgen für die Bewohner*innen, sondern hat spätestens im Zuge der Millennium-Entwicklungsziele auch zu einer Renaissance von Massenwohnungsbauprojekten, Verdrängungen und Umsiedlungen an den Stadtrand geführt. In Bezug auf Marokko zeigt der Artikel, inwieweit ein global verbreitetes, negatives Bild von Slums zu repressiven Wohnungspolitiken geführt hat. Aufbauend auf einer Haushaltsbefragung und qualitativen Interviews in einem sogenannten Slum in Casablanca dekonstruiert der Artikel bestehende Slum-Stigmata und zeigt, dass sich Slums nicht zwingend strukturell von anderen, gewöhnlichen Vierteln unterscheiden und maßgeblich durch Heterogenität gekennzeichnet sind. Als Konsequenz plädiert der Artikel dafür, Slums - analog zu Jennifer Robinsons Konzept der ordinary cities - als gewöhnliche Viertel zu bezeichnen und somit eine postkoloniale, empirisch-fundierte und vergleichend-analytische Sichtweise einzunehmen.

Ersteinreichung: 22. April 2020; Veröffentlichung online: 15. Dezember 2020

An English abstract can be found at the end of the document.

\section{Einleitung}

Slums werden oft als Inbegriff einer vermeintlich unterentwickelten Welt gesehen, als Sinnbild von fehl- oder ungesteuertem Wachstum der sogenannten Megastädte des globalen Südens. Nicht zuletzt durch apokalyptische Bücher wie Mike Davis' Planet der Slums (2007) hat sich ein Bild von Slums als Orte der Armut, der sozioökonomischen Ausgrenzung und katastrophaler Wohnbedingungen verfestigt. Wenngleich Davis seine Literaturarbeit auch mit struktureller Kritik an einer neoliberalen und neokolonialen Welt- und Wirtschaftsordnung verknüpft, so ist das in solchen Büchern und Berichten global verbreitete Image des Slums - mit seinen Bildern von Wellblechhütten an Berghängen oder in Überschwemmungsgebieten, neben Bahngleisen oder auf Müllkippen - längst zu einem bestimmenden Faktor entwicklungspolitischer Wohnungsdebatten geworden. Das Image des Slums gilt demnach als unvereinbar mit dem Leitmotiv ,erfolgreicher Entwicklung' und westlicher Moderne (Fischer 2014; Robinson 2002; Roy 2014; Valladares 2019). So wurde im Rahmen der Millennium-Entwicklungsziele (MDGs) das Ziel, den Anteil der in Slums lebenden Menschen weltweit zu reduzieren, zunächst mit dem Slogan ,Städte ohne Slums` verknüpft. Autor*innen wie Marie 
Huchzermeyer (2011a), Alan Gilbert (2007) und Pushpa Arabindoo (2011) haben eindrucksvoll beschrieben, wie die Rückkehr des stigmatisierten Begriffs ,Slum“ und der Slogan ,Städte ohne Slums“ eine neue Welle von Zwangsvertreibungen und Umsiedlungen ausgelöst hat. Damit verknüpft ist eine Rückkehr zu Massenwohnungs- und Umsiedlungsprogrammen in vielen Ländern des globalen Südens (Beier/Vilmondes Alves 2015; Buckley/Kallergis/Wainer 2016; Croese/Cirolia/Graham 2016; Turok 2016), deren standardisierte und primär quantitative Umsetzung Gefahr läuft, mehr Probleme zu verursachen als zu lösen (u. a. Beier 2019; Fleischer/Hurtado Tarazona/Alvarez Rivadulla 2019; Nikuze et al. 2019; Patel/Sliuzas/ Mathur 2015). In Anbetracht eines eklatanten Mangels an empirischen Daten über das Leben in ,Slums' (Turok/Borel-Saladin 2018) entstehen Wohnungsprogramme in vielen Ländern des Globalen Südens auf Basis von Grundannahmen, die weniger auf empirischer Evidenz als auf einer vorschnellen und externen Beurteilung der Lebensbedingungen in den betroffenen Siedlungen beruhen.

Ein typisches Beispiel ist das Programm Villes Sans Bidonvilles (VSB, auf Deutsch: Städte ohne Slums) in Marokko, dessen Ziel es ist, alle Slums in Marokko zu beseitigen. Dem globalen Image von Slums entsprechend werden bidonvilles[1] in Marokko als marginalisierte und desintegrierte Räume absoluter Armut stigmatisiert (Arrif 1999). Seit 2004 werden im Rahmen des größtenteils privat finanzierten VSB-Programms in über achtzig marokkanischen Städten Slums abgerissen und deren Bewohner*innen in neu entstehende Wohnsiedlungen am Stadtrand umgesiedelt. Den offiziellen Zielen des VSB-Programms zufolge soll das Programm urbane Armut reduzieren, Slumbewohner*innen in die Stadt integrieren und somit städtische Ausgrenzung bekämpfen (MHUPV 2012). Gleichzeitig steht das VSB-Programm im Zusammenhang mit Selbstmordattentaten von Slumbewohner*innen in Casablanca, die das bestehende Stigma von bidonvilles als Viertel der Ausgeschlossenen und als eine Brutstätte von religiösem Extremismus und Kriminalität verfestigten (Bogaert 2011). König Mohammed VI. hat Slums mehrfach als eine Bedrohung für die Entwicklung des Landes bezeichnet und betrachtet sie als unvereinbar mit seiner Vision eines wirtschaftlich starken, politisch stabilen, touristisch interessanten und modernen Marokkos (MHUPV 2013).

Dieser Artikel jedoch zeigt am Beispiel von Er-Rhamna, einem Slum in Casablancas Stadtteil Sidi Moumen, dass bei genauerer und vor allem empirischer Betrachtung von bidonvilles das stilisierte Image des Slums als Synonym von ,Unterentwicklung ins Wanken gerät. Die Gleichsetzung des Slums mit Armut und sozialer Ausgrenzung entbehrt zudem einer empirischen Grundlage. Stattdessen basiert das VSB-Programm auf einer externen Perspektive auf bidonvilles, die wesentlich durch Diskriminierung und Stigmatisierung beeinflusst wird. Aufbauend auf dieser empiriebasierten Argumentation hat der Artikel das konzeptionelle Ziel, Jennifer Robinsons (2006) Konzept der ordinary cities auf die Ebene der Stadtviertel zu übertragen. Es wird vorgeschlagen, Slums nicht länger als, besondere‘ oder ,rückständige‘ Orte einer Stadt, sondern vielmehr als gewöhnliche Viertel (ordinary neighbourhoods) zu betrachten, die sich dynamisch entwickeln und keine politische Sonderbehandlung benötigen. Die Gleichbehandlung als ordinary 
neighbourhoods ist notwendig, um die strukturelle Diskriminierung und Stigmatisierung von Slums zu bekämpfen und somit Stadtpolitiken entgegenzuwirken, die mit allen (repressiven) Mitteln ,Städte ohne Slums` errichten wollen. Wie andere gewöhnliche Stadtviertel auch, sollten Slums nicht auf Basis vorgefertigter, kolonial beeinflusster Stadtmuster, sondern auf Grundlage empirischer Evidenz und viertelspezifischer Konzepte entwickelt werden. Der Artikel ruft zu mehr empirischen und vergleichenden Arbeiten zu Lebensweisen und Lebensbedingungen in Slums auf.

Der Forschung zu diesem Artikel liegt ein Mixed-Methods-Ansatz zugrunde, dessen Kern eine repräsentative Haushaltsumfrage mit 401 systematisch ausgewählten Bewohner*innen Er-Rhamnas bildet.[2] Der Fragebogen sammelte nicht nur quantitative Daten zu Themen wie Wohnqualität und -zufriedenheit, Nachbarschaft, Mobilität und Einkommen, sondern wurde, wenn die befragten Personen gewillt waren, mehr zu erzählen, wie ein semistrukturierter Interview-Leitfaden verwendet. In etwa einem Drittel der Fälle konnten so qualitative Interviews von bis zu zwei Stunden Länge zu individuellen Schwerpunktthemen geführt werden. Die Interviews wurden in Darija[3] geführt, was von allen kontaktierten Personen fließend gesprochen wurde. Der Autor war während der Umfrage vor Ort und führte die Interviews zusammen mit zwei studentischen, frankophonen Übersetzer*innen (m/w) durch, die beide keinen Bezug zu Er-Rhamna hatten. Beide notierten über die Fragenbögen hinaus Anmerkungen und Kommentare der Befragten, welche es dem Autor ermöglichten, flexibel Rückfragen zu stellen. Jedes Interview wurde anschließend mit den Übersetzer*innen besprochen, um sprachliche Unklarheiten zu beseitigen.

Im nun folgenden 2. Abschnitt beschreibt der Artikel zunächst die Grundzüge des Konzepts der ordinary cities (Robinson 2006) und nimmt Bezug auf internationale Leitmotive der Entwicklungspolitik in Bezug auf Slums. Dabei wird auf eine global verbreitete Stigmatisierung von Slums eingegangen, die Wohnungsbau- und Umsiedlungspolitiken geprägt hat und dabei mehr auf Annahmen denn auf empirischer Evidenz beruht. Der Artikel nimmt dabei im Besonderen Bezug auf das marokkanische VSBProgramm. Der nächste Abschnitt (3.) des Artikels analysiert auf Basis der Empirie die Lebensbedingungen und Lebensweisen von Bewohner*innen in Er-Rhamna und kontrastiert sie mit bestehenden Stigmata. Im Fazit (4.) werden Wege aufgezeichnet, wie Slums analog zu Robinson (2006) als ordinary neighbourhoods bezeichnet werden können und welche Forderungen sich daraus für die Stadtentwicklungsforschung ableiten.

\section{Slums zwischen Megastadtsymbolik, global und ordinary cities}

„Rather than categorising and labelling cities as, for example, Western, Third World, developed, developing, world or global, I propose that we think about a world of ordinary cities, which are all dynamic and diverse, if conflicted, arenas for social and economic life." (Robinson 2006: 1)

In wenigen Worten prägnant zusammengefasst, beschreibt dieses Zitat von der ersten Seite des Buchs Ordinary Cities das Grundprinzip hinter Jennifer 
Robinsons Konzept der gewöhnlichen Städte. Das Ziel der Autorin ist die Entwicklung einer vergleichenden, postkolonialen Perspektive auf „Städte in einer Welt aus Städten“ (vgl. Robinson 2016a, 2016b), die sich loslöst von dominanten Kategorien und Hierarchien, die die Städte der Welt spätestens seit den 1980er Jahren grob in zwei Lager unterteilen: Auf der einen Seite stehen die global cities oder world cities, die, zurückgehend auf Saskia Sassen (2001) und John Friedmann (1986), als die ,modernen“ wirtschaftlichen Zentren der Globalisierung bezeichnet werden können. Auf der anderen Seite stehen die Megastädte des globalen Südens mit ihrem vermeintlich unkontrollierten Wachstum, dessen Symbol - der Slum - als Antithese der ,modernen' Stadt ein Bild der Rückständigkeit zeichnet und Fragen der „Entwicklung' aufwirft (vgl. Roy 2011). Während sich wesentliche Theorien der Stadt(-entwicklung) wie selbstverständlich mit universellem Anspruch in wenigen Städten des globalen Nordens konstituiert haben, werden die Megastädte des globalen Südens durch Theorien der (Unter-)Entwicklung erklärt. Daraus ergibt sich ein hegemonialer Anspruch westlicher Stadttheorie und -moderne, der kaum infrage gestellt wird (vgl. Lawhon/Truelove 2020; McFarlane 2008; Watson 2009). Es wirkt normal, dass Städte wie Mumbai oder Rio de Janeiro sich an der Moderne New Yorks orientieren und messen. Umgekehrt erscheint ein solcher Vergleich ungewöhnlich. Mumbai und Rio de Janeiro werden scheinbar automatisch mit Bildern von Favelas und Slums verknüpft, während die Glastürme von New York implizit als Sinnbild der Moderne und der global city gelten. Robinsons Ziel ist es, mithilfe des Konzepts der ordinary cities die Lücke zwischen global cities und megacities $\mathrm{zu}$ überwinden und eine neue, kritische Stadtforschung auf der Basis von unvoreingenommenen Vergleichen zwischen Städten zu etablieren. Deren Ziel sollte es sein, klassische Annahmen in Bezug auf Stadt (im Singular) zu hinterfragen, um neue, postkoloniale Theorien von Städten (im Plural) zu generieren (Robinson 2016a, 2016b). In den letzten Jahren hat die postkoloniale Stadtforschung an Bedeutung gewonnen (Lawhon et al. 2020; Lawhon/Truelove 2020; Schindler 2017) und sich unter anderem verstärkt der Entwicklung sozial konstruierter Stigmata von ,Megastadt‘ und „Slum ' (Roy 2011; Fischer 2014; Valladares 2019) sowie der daraus resultierenden Überhöhung vermeintlicher Unterschiede zwischen ,formellen“ und ,informellen' Stadträumen gewidmet (Cavalcanti 2014; Meth 2020; Yiftachel 2009). Hierauf soll im Folgenden näher eingegangen werden, bevor ich im letzten Abschnitt dieses Artikels wieder an das Konzept der ordinary cities anknüpfe.

Um die Jahrtausendwende erlebte der Begriff ,Slum ‘ auf internationaler Ebene eine Renaissance (Gilbert 2007; Huchzermeyer 2013). Die Initiative Cities Alliance verkündete ihren Slogan „Städte ohne Slums“ (World Bank/ UN-Habitat 2000), der später in die MDGs übernommen wurde. UNHabitat (2003) veröffentlichte den Bericht The Challenge of Slums und Mike Davis (2007) schürte mit seinem Buch Planet der Slums Horrorvisionen. Dabei wurde der ,Slum ' zum Symbol der ,unterentwickelten' Megastadt, von Armut, Ausgrenzung und Ausbeutung - unvereinbar mit dem Ideal slumfreier und somit ,entwickelter', ,moderner' und international wettbewerbsfähiger Städte. Slums werden dabei nicht nur als kriminelle No-Go-Areas und als das Ergebnis einer Invasion von Landflüchtigen bezeichnet. Ihren 
Bewohner*innen wird oft jegliche,legitime‘ Stadtzugehörigkeit abgesprochen (Bogaert 2011; Cavalcanti 2014; Husseini de Araújo/Batista da Costa 2017; Valladares 2019).

In diesem Zusammenhang befürchtete Alan Gilbert (2007: 701), dass das negative Image des ,Slums ' zu neuen politischen Kampagnen, Vertreibungen und Programmen führen würde, die Slumbewohner*innen stärker stigmatisieren und deren Lebensbedingungen nicht verbessern, sondern gefährden würden. Bereits James A. Yelling (1986: 1) stellte fest, dass der Begriff ,Slum eine Verurteilung bestehender Verhältnisse und einen Handlungsaufruf impliziere. Zwänge des internationalen Städtewettbewerbs haben diesen Handlungsaufruf weiter verstärkt (Huchzermeyer 2011a; Roy 2014). Wenn sich Städte als ,modern ' und ,entwickelt' auf dem Weltmarkt vermarkten wollen, dann versuchen sie, Slums zu verstecken oder zu beseitigen. Im Zuge von internationalen Megaevents wurden beispielsweise in Brasilien und Südafrika verstärkt Slums abgerissen und Bewohner*innen umgesiedelt (Steinbrink/ Haferburg/Ley 2011; Steinbrink et al. 2015). In Marokko wurden bidonvilles in der Einflugschneise des internationalen Flughafens von Casablanca nicht saniert, sondern umgesiedelt, weil Politiker*innen befürchteten, internationale Gäste könnten beim Anblick auf die ärmlichen Hütten aus dem Flugzeug ein schlechtes Bild von Marokko erhalten (Arandel/Wetterberg 2013: 143). Selbst wenn Slums nicht abgerissen, sondern aufgewertet werden, werden Stigmata von Andersartigkeit, Kriminalität und Armut konserviert und mitunter gar von touristischen Stadtmarketingstrategien vereinnahmt (Roy 2011; Valladares 2019).

Eng verknüpft mit der Rückkehr des ,Slums“ in die internationale politische Debatte sind die MDGs. Die MDGs fokussierten sich in ihrem einzigen explizit stadtbezogenen Ziel auf das Symbol städtischer ,Unterentwicklung6 - die Slums. Sie machten die Entwicklungsforschung und -politik zu einem bedeutenden Treiber der Stadtentwicklung im globalen Süden und legitimierten so die Vormachtstellung der slumfreien Städte des Nordens, die keine Entwicklung mehr benötigten. Die MDG-Zielvorgabe 7.D lautete: „Bis 2020 eine erhebliche Verbesserung der Lebensbedingungen von mindestens 100 Millionen Slumbewohnern herbeiführen“(BMZ o. J.). Anfangs wurde dieses Ziel wörtlich mit dem Slogan „Städte ohne Slums“ der Cities Alliance verbunden (Huchzermeyer 2013: 301) und weitergehend „sorglos mit [ihm] vermengt“ (Berner 2016: 10). Obwohl Cities Alliance und die MDGs participatory slum upgrading als bevorzugten Politikansatz propagierten, setzten sie mit dem unvorsichtig formulierten Slogan doch implizite Anreize für slum eradication, also eine rein quantitative Reduzierung von Slums. Slum eradication wurde so zu einem legitimen Teil vermeintlicher Entwicklung und konnte von den Regierungen mit ihren MDG-Anstrengungen gerechtfertigt werden (Arabindoo 2011; Huchzermeyer 2013). Diese Anreize sind bis heute auch in den Indikatoren der nachfolgenden Ziele für nachhaltige Entwicklung (SDGs) präsent. Das SDG-Unterziel 11.1, ,Zugang zu angemessenem, sicherem und bezahlbarem Wohnraum [sicherzustellen] und Slums [zu] sanieren“, wird gemessen durch den „Anteil der urbanen Bevölkerung, die in Slums, informellen Siedlungen oder unangemessenen Unterkünften lebt" (Statistisches Bundesamt 2020). Der Indikator setzt einen falschen Anreiz für die Zerstörung von Slumsiedlungen und die Vertreibung von 
Slumbewohner*innen, um eine positive Veränderung des Indikators zu erreichen. Dem oben zitierten, eigentlichen Ziel von SDG 11.1, Wohnsituationen zu verbessern, läuft dies klar zuwider.

Nichtsdestotrotz entstanden in den vergangenen Jahren immer mehr, größtenteils privat finanzierte Massenwohnungsbauprogramme, die auf eine quantitative Reduktion von Slumbewohner*innen abzielen, ohne sich genauer mit den Ursachen von und Lebensbedingungen in Slums auseinanderzusetzen (Buckley/Kallergis/Wainer 2016; Croese/Cirolia/ Graham 2016; Turok 2016). Vielmehr stellen sie die Materialisierung eines politisch-planerischen Schwarz-weiß-Verständnisses ,informeller und ,formeller 'Stadträume dar (Meth 2020). Dieser Trend ist nicht allein den MDGs und der Renaissance des Slum-Begriffs zuzuschreiben. Zahlreiche Länder haben jedoch neue großmaßstäbliche und repressive Programme zur Bekämpfung von Slums mit direktem Bezug zu den MDGs ins Leben gerufen (Arabindoo 2011; Huchzermeyer 2013). Marokkos Regierung betrachtet zum Beispiel das VSB-Programm, welches direkten wörtlichen Bezug auf „Städte ohne Slums“ nimmt, als eine Umsetzungsmaßnahme der MDGs (MHUPV 2012: 14). Gleichzeitig ist in Casablanca das VSB-Ziel, ,Städte ohne Slums ' zu erreichen, verbunden mit der strategischen Vision, Casablanca zu einer internationalen Finanzmetropole auszubauen. Zudem nutzt der Staat das VSBProgramm dazu, zentrale, lukrative Flächen von bidonvilles gegen periphere öffentliche Grundstücke einzutauschen. Das VSB-Programm kann somit als eine neoliberale Umsetzung eines westlichen Entwicklungsverständnisses verstanden werden (vgl. Bogaert 2018), das im Zuge von Cities Alliance, MDGs und SDGs das Image von Slums als Antithese von Entwicklung und als ein Symbol von Rückständigkeit manifestiert hat.

Bilder von ,hässlichen', ,rückständigen' Slums passen nicht zum Weltklasse-Image, das Großprojekte wie die neue Waterfront „Casablanca Marina" vermarkten sollen, um Investor*innen und Tourist*innen anzulocken (Bogaert 2018: 2). König Mohammed VI. bezeichnete 2001 das Fortbestehen von bidonvilles in Marokko als eine Gefahr für die wirtschaftlichen Entwicklungserfolge des Landes (MHUPV 2013: 49). Das bezieht sich nicht nur auf störende Bilder von ,armseligen“ und ,unmodernen“ Hüttensiedlungen, sondern auch auf das Stigma von bidonvilles als Horte der Kriminalität und des Extremismus - aber auch der sozialen Revolte (Beier 2018; Bogaert 2011; Zaki 2005: 52 ff.). In seinen Reden hat sich König Mohammed VI. geschickt als König der Armen präsentiert, der nicht die Bewohner*innen der bidonvilles selbst, sondern die zugrundeliegenden Strukturen von Ausgrenzung und Armut in den bidonvilles verurteilt, welche deviantes Verhalten fördern würden. Das VSB-Programm soll demnach bidonvilles als „Quellen von Frustration, Exklusion, Devianz und Extremismus“ (König Mohammed VI. in MHUPV 2013: 68; Übers. d. A.) beseitigen und ihre Bewohner*innen durch Umsiedlung in neue Wohnungen in die Stadt integrieren. In den neuen Wohnvierteln am Stadtrand sollen deren Kinder ,zu guten Stadtbürger*innen von morgen heranwachsen“ (König Mohammed VI. in MHUPV 2013: 123; Übers. d. A.).

Inwieweit bidonvilles jedoch als Orte der Ausgrenzung und Armut bezeichnet werden können, ist ebenso fraglich wie die Annahme, dass Umsiedlungen Armut und Ausgrenzung reduzieren können (Bartoli 2011; Beier 2019). 
Abdelmajid Arrif ist überzeugt, dass solche „externen Sichtweisen“ mehr über ihre Betrachter*innen verraten als über den Lebensraum bidonville“ (1999: 299; Übers. d. A.). Das VSB-Programm gründet zu großen Teilen auf Annahmen und Behauptungen, die auf einer solchen Außenperspektive beruhen (Beier 2019: 133). Generell fehlt es an empirischer Forschung zu Anzahl, Lebensbedingungen und Lebensweisen von Slumbewohner*innen (Deboulet 2016; Huchzermeyer 2011a: 77) - insbesondere in weniger bekannten Siedlungen (Valladares 2019). Auch mit Blick auf die Wirkung von Slums auf soziale Mobilität fehlt es an empirischer Evidenz (Turok/BorelSaladin 2018). Der folgende Abschnitt, im Grundprinzip dem theoretischen Ansatz von Robinson (2006: 2) folgend, hinterfragt daher kritisch Grundannahmen hinter dem ,Slum' als Symbol der megacities.

\section{Realitäten versus Stigma - ein empirischer Blick auf Er-Rhamna}

„Was der Unterschied ist zwischen der ancienne médina [Altstadt][4] und Er-Rhamna? Nichts! Außer vielleicht, dass man hier in Er-Rhamna besser leben kann." (Mohammed[5], 28, Straßenverkäufer in Casablancas medina und Bewohner von Er-Rhamna)[6]

Von außen mögen diese beiden Siedlungsräume sich fundamental unterscheiden: auf der einen Seite die medina - das Symbol der ,orientalischen Stadt'[7] und anerkanntes Weltkulturerbe in mehreren marokkanischen Städten -, auf der anderen Seite das bidonville oder der Slum - das Symbol für ,Unterentwicklung' und Armut. Während die medina als Aushängeschild marokkanischer Kultur vermarktet wird, wird das bidonville als Gefahr für den Tourismus gesehen (König Mohammed VI. 2001 in MHUPV 2013: 49). Nimmt man jedoch, wie mein Interviewpartner Mohammed, die Innenperspektive ein, verschwimmen vermeintlich klare Unterschiede. So betont Arrif (1999: 301) die Ähnlichkeit von bidonvilles mit dem klassischen Modell der arabisch-islamischen Stadt, wie sie die medina verkörpert. Zudem war letztere klassischerweise ein Wohnraum ökonomisch schwächerer Bevölkerungsschichten, gekennzeichnet durch marode Bausubstanz und veraltete Infrastruktur (Benlahcen Tlemçani/ Missamou 2000). Das touristische Potential der medina hat in den vergangenen Jahrzehnten jedoch verstärkt ausländisches und inländisches Kapital angezogen. Historische Bausubstanz wurde renoviert, Basisinfrastruktur erneuert und touristische Infrastruktur ausgebaut. In Städten wie Marrakesch hat dies auch zu Gentrifizierung geführt (Escher/Petermann/Arnold 2018; Spotorno 2019). In weniger touristischen Städten wie Casablanca und Salé blieben diese Aufwertungs- und Verdrängungsprozesse weitestgehend aus. Teile von Casablancas Altstadt wurden gar abgerissen, um Platz zu schaffen für den geplanten Bau eines Prachtboulevards (Berry-Chikhaoui 2010).

In meinen eigenen Vorträgen und Seminaren innerhalb und außerhalb Marokkos habe ich mehrfach eine Art Rätsel verwendet, in dem ich die Teilnehmenden bitte, fünf durcheinandergewürfelte Bilder aus der Altstadt der UNESCO-Weltkulturerbe-Stadt Rabat und aus Casablancas bidonville ErRhamna der jeweiligen Siedlung zuzuordnen. Wenige haben bislang alle fünf 


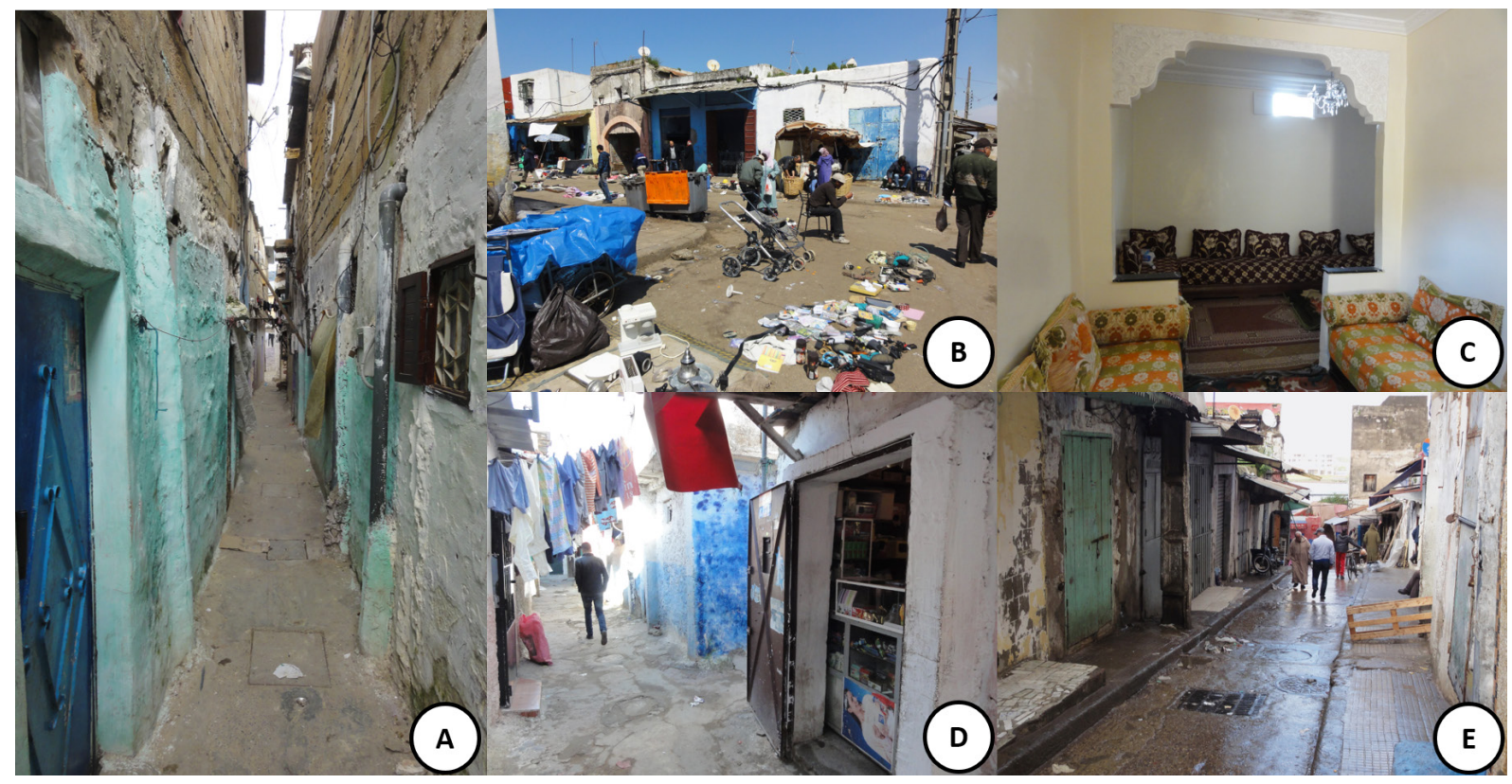

Bilder richtig zugeordnet (vgl. Abb. 1). Dennoch: Kaum jemand hinterfragt, dass in Marokko medinas renoviert und touristisch vermarktet werden, während bidonvilles im ganzen Land „ausgerottet“ (éradiquer; MHUPV 2012: 13) werden sollen. Selbstverständlich stehen die bewusst ausgewählten Bilder weder für Er-Rhamna noch für Rabats Altstadt im Allgemeinen. Das Rätsel aber offenbart vorgefertigte Images und Verallgemeinerungen, die zu reflektieren sind.

Er-Rhamna ist eines der letzten bidonvilles in Casablancas Stadtteil Sidi Moumen, welcher als Heimat der Selbstmordattentäter von 2003 unrühmliche Bekanntheit erlangte. Mit etwa 37.00o Einwohner*innen in etwa 6.00o Häusern auf einer Fläche von lediglich 27 Hektar ist es das größte noch bestehende bidonville Marokkos und wurde deshalb für die hier vorgestellte Studie ausgewählt. Seit Jahren hören die Bewohner*innen regelmäßig, dass sie sehr bald umgesiedelt werden, doch die Umsetzung des VSB-Programms lässt in Er-Rhamna weiter auf sich warten.[9]

Das einleitende Beispiel zeigt, wie wichtig es ist, die Perspektive zu wechseln und Wohnbedingungen nicht nur aus der Außen-, sondern vor allem aus der Innen- und somit der Bewohner*innenperspektive zu bewerten (vgl. Abb. 2) (vgl. Geiselhart 2017: 228; Perlman 2016). Während das Stigma von Armut und ,Unterentwicklung' vor allem auf der Außenperspektive beruht, wird aus der Innenperspektive eine größere Heterogenität der Lebensbedingungen deutlich. Armut und prekäre Lebensverhältnisse bleiben auch der Innenperspektive nicht verborgen; sicherlich würden nicht alle Bewohner*innen Er-Rhamnas Mohammeds Vergleich mit der medina vom Beginn des Kapitels zustimmen. Die Innenperspektive verhindert aber eine vorschnelle Verallgemeinerung, die mit stigmabelasteten Begriffen wie ,Slum ' und ,bidonville einhergeht. Soziologiestudentin und Bewohnerin Nadia, 28, betont:

„Du kannst nicht sagen, wo du herkommst! Sagst du, du kommst aus dem bidonville, gucken dich die Leute schief von der Seite an. Viele, die so reagieren, sind noch nie hier gewesen. Aber wenn du von außen die Viertel und die Leute bewertest, dann ist das oberflächlich!“
Abb. 1 Welche Fotos zeigen die Altstadt von Rabat und welche den Slum Er-Rhamna in Casablanca? (Quellen: eigene Fotos[8]) 


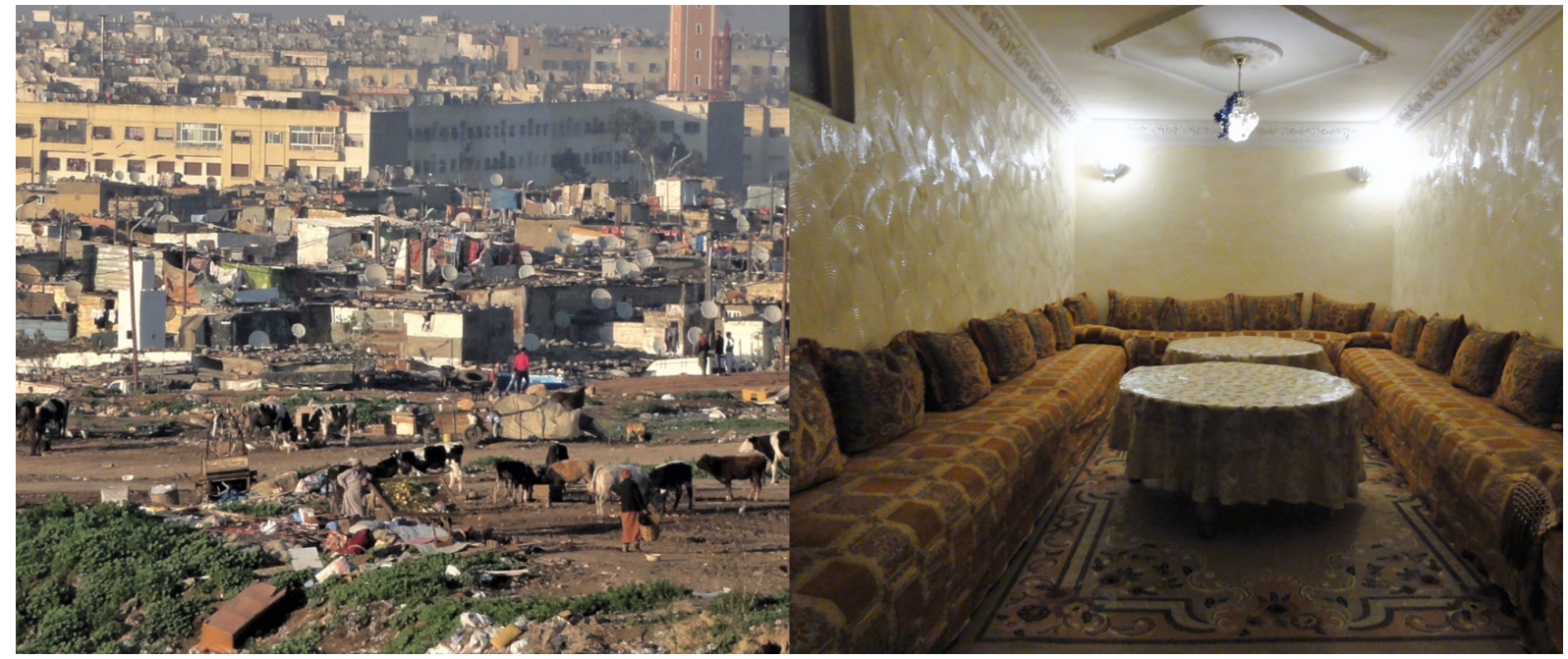

Abb. 2 Gegensätzliche Außen- und Innenansichten der Wohnbebauung in ErRhamna, Casablanca (Quellen: eigene Fotos, Februar und März 2017)
Im Fall von Er-Rhamna wird dies auch dadurch verstärkt, dass die Siedlung von innen nach außen gewachsen ist. Das heißt, ältere und über die Jahre ausgebaute, verstetigte und verschönerte Häuser befinden sich vor allem im Inneren der Siedlung, während jüngere, oft prekärer aussehende Häuser überproportional oft an den Rändern der Siedlung liegen und somit stärker von außen wahrgenommen werden können.

Das Stigma des ,Slums“ hat in Er-Rhamna erheblichen Einfluss auf das Leben der Bewohner*innen und auf eine im Durchschnitt positive Haltung zu einer möglichen Umsiedlung an den Stadtrand. Nachhilfelehrer Khalid, 21, meint dazu:

„Manche Bewohner*innen würden sogar eine Umsiedlung akzeptieren, wenn sie zum Mond ziehen müssten. Viele sehen die Probleme nicht, die auf sie zukommen. Aber ein großes Problem ist die Stigmatisierung. Seit der Schule bekommen die Leute hier zu hören, dass sie Menschen zweiter Klasse sind. [...] Und wenn dich jemand aufgrund deines Wohnorts beleidigt und du dich wehrst, hat er immer bessere Karten. Er kann die Polizei rufen und keiner wird dir glauben, weil du im karyan[10] wohnst.“

Eine andere Bewohnerin schilderte, dass sie jedes Mal, wenn sie ein Taxi benutzt, sich nicht traut, dem Fahrer zu sagen, dass er sie nach Er-Rhamna bringen soll. Sie lässt sich dann ins benachbarte Viertel Saâda fahren und läuft nach Hause. Umgekehrt steuern manche Taxifahrer bidonvilles aus Angst nicht an (Zaki 2005: 63).

Im Allgemeinen beruht die Stigmatisierung der bidonvilles in Marokko nicht nur in Er-Rhamna - auf verschiedenen, grob differenzierbaren Einzelaspekten. Laut Lamia Zaki (ebd.: 49 ff.) zählen dazu:

- die ästhetische Stigmatisierung in Bezug auf die Bausubstanz,

- die sozio-ökonomische Herabwürdigung und das Absprechen von agency,

- das Abstreiten der Stadtzugehörigkeit (urban citizenship),

- die Kriminalisierung der Bewohner*innen als ,illegale Schmarotzer*innen' und

- die Brandmarkung als politische Unruheherde und Brutstätten des Extremismus. 
Die genannten Aspekte stehen in gegenseitiger Wechselwirkung und sind zudem auch in anderen geographischen Kontexten häufig Bestandteile der Stigmatisierung von Slums (vgl. Bayat/Denis 2000; Geiselhart 2017; Ghertner 2012; Gilbert 2007; Holston/Caldeira 2008; Husseini de Araújo/ Batista da Costa 2017; Perlman 2016). Um das typische Stigma des ,Slums zu dekonstruieren, werde ich im Folgenden mit Ausnahme des letzten Punkts (vgl. hierfür Beier 2018; Zemni/Bogaert 2011) auf alle oben genannten Einzelaspekte eingehen und diese, basierend auf selbst erhobenen Daten zu Er-Rhamna, kritisch hinterfragen.

\subsection{Wohn- und Infrastrukturqualität in Er-Rhamna}

Das für das VSB-Programm verantwortliche Ministerium definiert ein $b i$ donville als „eine Barackensiedlung, bestehend aus notdürftigen Hütten, oft errichtet mit einfachen und verschiedenartigen Recyclingmaterialien (z. B. Wellblech und Holz) auf Flächen ohne jegliche Basisinfrastruktur (Abwasser, Trinkwasser, Strom...)“ (MHUPV 2012: 12; Übers. d. A.). Diese Definition spiegelt das typische Image von Slums wider und setzt sie mit Unterentwicklung und Rückständigkeit gleich. Es zeigt auf deutliche Weise die staatlich gelenkte Stigmatisierung von bidonvilles und entspricht in keiner Weise der Realität in Marokko. Typisch für viele informelle Siedlungen und den Theorien John F. C. Turners (1977) entsprechend wurde Er-Rhamna durch seine Bewohner*innen über mehrere Jahrzehnte hinweg inkrementell entwickelt und verstetigt. Das Viertel entstand Ende der 1960er Jahre durch Migrant*innen aus der Provinz Rhamna nahe Marrakesch, die sich zunächst in Zelten auf einer großen Brachfläche neben einem alten Steinbruch niederließen. Laut Bewohnerin Hafida zahlte ihr Vater, als er aus der Sahara in den 1970er Jahren nach Casablanca kam, 40 Dirham an den qaïd[11] für ein Stück Land, auf dem er eine Holzhütte errichten durfte. Über die Jahre hinweg hat er mehrfach den qaïd um Erlaubnis gefragt (und bezahlt), um sein Haus ausbauen zu dürfen. Heute hat es zwei Etagen und drei Schlafzimmer mit insgesamt etwa 6o Quadratmetern Wohnfläche für drei kleine Haushalte. Zahlreiche weitere Bewohner*innen haben, durchaus mit Stolz, ähnliche Geschichten über ihren familiären Hausbau berichtet.

Heute bestehen nahezu alle Häuser in Er-Rhamna aus Stein und Zement, 28 Prozent der Häuser haben zwei oder drei Etagen und im Schnitt beträgt die Wohnfläche 50 bis 60 Quadratmeter. Alle Häuser besitzen einen formellen Stromanschluss[12] und eine eigene Toilette und 95 Prozent verfügen über einen Wasseranschluss im Haus. Auf der anderen Seite schützt das Dach von nur einem Viertel der Häuser immer und effektiv vor Regen und ist in über 90 Prozent der Fälle aus Wellblech. Der Wasserdruck ist vor allem im Sommer oft niedrig und über Stromausfälle sowie Feuer als Folge von Kurzschlüssen wird regelmäßig berichtet. Da die Siedlung nicht an das Kanalsystem angeschlossen ist, müssen Abwassertanks im Boden regelmäßig durch die Bewohner*innen selbst oder beauftragte Klempner geleert werden.

Was die Zufriedenheit mit Infrastruktur und dem eigenen Haus angeht, ergibt sich ein gespaltenes Bild, das die Heterogenität von Er-Rhamna unterstreicht. Insgesamt sind nahezu gleich viele Personen zufrieden (44 Prozent) wie unzufrieden (41 Prozent) mit der Infrastruktur. Was die Haus- und 
Tab. 1 Pearson-Korrelationen zwischen Pro-Kopf-Einkommen bzw. Wohndauer und Wohnqualität und -zufriedenheit in Er-Rhamna (Quelle: eigene Daten)

\begin{tabular}{|c|c|c|c|c|}
\hline Einheit & $\begin{array}{c}\text { Größe des } \\
\text { Hauses }\end{array}$ & Wohndichte & $\begin{array}{c}\text { Das Dach } \\
\text { schützt vor } \\
\text { Regen }\end{array}$ & $\begin{array}{c}\text { Zufriedenheit } \\
\text { mit dem Haus }\end{array}$ \\
\hline $\begin{array}{c}\text { Pro-Kopf- } \\
\text { Einkommen } \\
\text { des Haushalts } \\
\text { (Dirham/Kopf) }\end{array}$ & $\begin{array}{c}\text { Haushalts- } \\
\text { mitglieder pro } \\
\text { Raum }\end{array}$ & $j$ ja/nein & $\begin{array}{c}\text { 5-stufige, } \\
\text { aufsteigende } \\
\text { Likert-Skala }\end{array}$ \\
\hline $\begin{array}{c}\text { Wohndauer } \\
\text { (Jahre) }\end{array}$ & $\mathbf{0 , 2 0 9 * * *}$ & $\begin{array}{c}-\mathbf{0 , 2 6 4 * * *} \\
(\mathrm{n}=380)\end{array}$ & $\begin{array}{c}\mathbf{0 , 1 4 8 *} \\
(\mathrm{n}=369)\end{array}$ & $\begin{array}{c}\mathbf{0 , 1 0 8 *} \\
(\mathrm{n}=380)\end{array}$ \\
\hline
\end{tabular}

Signifikanzniveaus: $* p \leq 0,05 ; * * p \leq 0,01 ; * * * p \leq 0,001$

Wohnqualität angeht, so ist ein Drittel der Haushalte zufrieden, während 40 Prozent mit ihrem Haus unzufrieden sind. Die Daten der Haushaltsbefragung zeigen außerdem, dass Einwohner*innen ihr Einkommen nutzen, um in ihr Haus zu investieren. Je höher das Pro-Kopf-Einkommen der Haushalte ist, desto größer das Haus, desto niedriger die Wohndichte (Haushaltsmitglieder pro Raum) und desto wahrscheinlicher schützt das Dach vor Regen. Auch die Zufriedenheit mit dem Haus steigt mit höherem Pro-Kopf-Einkommen (vgl. Tab. 1). Zudem korreliert die Wohndauer signifikant positiv mit der Hausgröße sowie der Zufriedenheit mit dem Haus, was nahelegt, dass Personen über die Zeit ihre Häuser ausbauen (vgl. Tab. 1). Allerdings könnten die Investitionen in das Haus und somit auch der Wohnkomfort vermutlich deutlich größer sein, würden nicht Gerüchte über eine baldige Umsiedlung die Investitionsbereitschaft hemmen. Bouchra, 28, erzählte, dass sie eine alte Werkstatt in Er-Rhamna ausgebaut hat und mit ihrem Mann dort eingezogen ist:

„Einige hier haben durchaus Erspartes, aber sie investieren es nicht in die Pflege, den Ausbau oder die Renovierung ihrer Häuser, weil sie jederzeit damit rechnen, umgesiedelt zu werden. Ich sehe das nicht so. Ich finde, man sollte es sich im Hier und Jetzt schön machen.“

Er-Rhamna hat sich also über Jahrzehnte hinweg entwickelt und verbessert. Der Staat hingegen hat seit den 200oer-Jahren durch die wiederholten Gerüchte über eine nahende Umsiedlung diese Entwicklung aktiv gebremst. Das zeigt sich auch bei den Umzugsaktivitäten: Auch Haushalte, die es sich leisten könnten und die willens sind, auszuziehen, bleiben in Er-Rhamna wohnen, um nicht den Anspruch auf eine Wohnung vom Staat zu verlieren. Die natürliche Dynamik und Fluktuation des Viertels sind bedingt durch Gerüchte und verstärkte staatliche Kontrolle zum Erliegen gekommen.

\subsection{Armut in Er-Rhamna}

Wie bereits beschrieben, sieht der marokkanische Staat das VSB-Programm als wichtigen Beitrag zum Kampf gegen städtische Armut und Ausgrenzung. Laut des federführenden Ministeriums „stellen bidonvilles eine Brutstätte par excellence für Armut und soziale Ausgrenzung im urbanen Raum dar“ 
(MHUPV 2012: 33; Übers. d. A.). Zaki (2005: 62) betont, dass das Außenbild des Slums als armseliger und marginalisierter Raum nahtlos auf die Bewohner*innen übertragen wird. Ein Blick auf die Daten der Haushaltsumfrage zeigt jedoch, dass - beruhend auf nationalen Armutsgrenzen (HCP 2018) ,nur 15 Prozent der Haushalte als ,monetär arm` zu klassifizieren sind und somit über ein Pro-Kopf-Jahreseinkommen von weniger als 4.668 Dirham (ca. 420 Euro) verfügen. Eine Anfälligkeit für Armut und somit ein ProKopf-Jahreseinkommen zwischen 4.668 und 7.001 Dirham (ca. 630 Euro) betrifft weitere 28 Prozent der Haushalte (vgl. Abb. 3). Diese Werte liegen zwar deutlich über der methodisch bedingt schwer zu vergleichenden offiziellen Armutsrate in Sidi Moumen (5,6 Prozent arm oder armutsanfällig, HCP 2018), sie zeigen aber auch, dass über die Hälfte der Haushalte in ErRhamna weder arm noch armutsanfällig sind.

Auf Basis der Interviews mit Bewohner*innen lassen sich zudem mindestens drei Gründe dafür feststellen, dass monetäre Armut in bidonvilles wie Er-Rhamna erträglicher ist als in anderen städtischen Vierteln, und zwar aufgrund geringer Wohnkosten, starker Solidaritätsnetzwerke und der relativ zentralen Lage. Viele Interviewte gaben als Vorteil eines Lebens im bidonville an, dass sie keine Miete zahlen müssen. Insbesondere für Haushalte mit schwankenden Einkommen bedeutet das Leben im bidonville daher mehr finanzielle Sicherheit und oft auch mehr Wohnfläche. Beispielhaft erzählte eine 36-jährige Bewohnerin: „Als wir in Hay Hassani wohnten, hatte mein Vater es weit zur Arbeit und wir hatten nicht immer das Geld für die Miete." In einem anderen Fall berichtete eine Putzkraft glücklich, dass ihr Chef ihr vor einiger Zeit ein Haus in Er-Rhamna für 43.00o DH (ca. 3.900 Euro) gekauft hatte. Nachdem ihr Mann chronisch krank und arbeitsunfähig geworden war, hatte sie zuvor kein Geld mehr gehabt, um neben den Medikamenten auch Wasser und Strom bezahlen zu können. Im Falle von Armut helfen zudem familiäre und nachbarschaftliche Solidaritätsnetzwerke, die am häufigsten als Vorteil des Lebens in Er-Rhamna genannt und als eine Kultur der Arbeiterklasse beschrieben wurden (vgl. Arrif 1999: 311; Beier 2019: 151 ff.). „Das Sozialleben ist hier nicht wie in Mietswohnhäusern, wo jede Tür geschlossen ist“, sagte ein 45-jähriger Nachtwächter. Eine 80-jährige Bewohnerin fasste es prägnant zusammen. Auf die Frage, was sie im Viertel schätzte, antwortete sie: „Meine Nachbarn! In 40 Jahren haben wir niemals gehungert!“ Als dritter armutsmildernder Faktor ermöglicht es die Lage von Er-Rhamna aufgrund

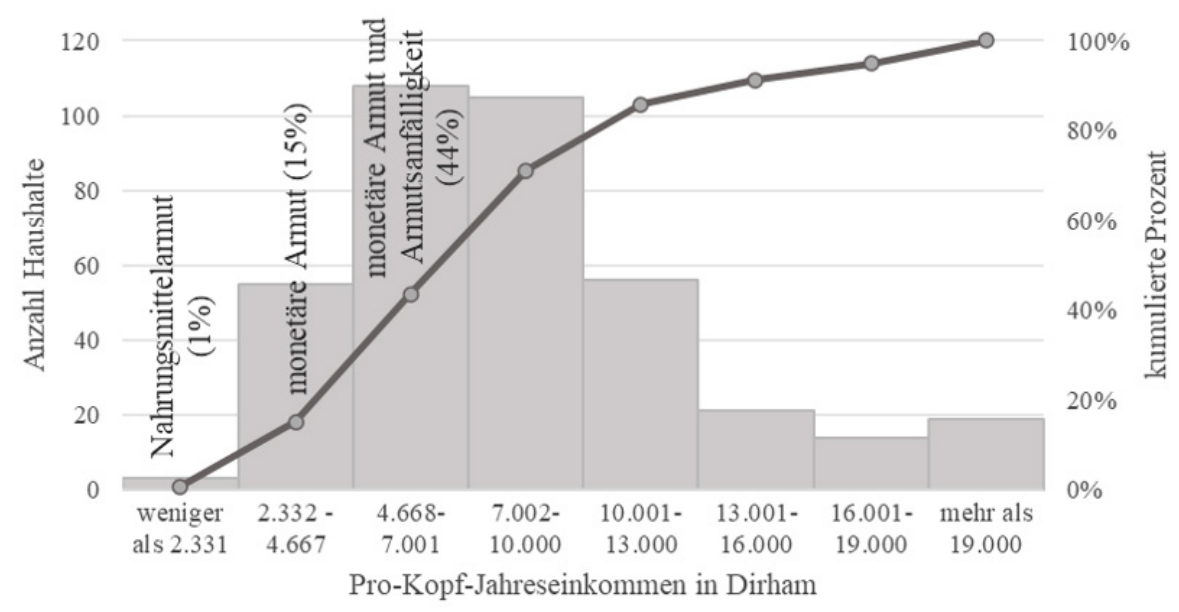

Abb. 3 Histogramm und kumulierte Verteilung von Pro-KopfJahreseinkommen und Armut in Er-Rhamna ( $n=381$; Quelle: eigene Daten) 
seiner Nähe zu vielen Arbeitsplätzen, Transportkosten zu sparen und auch kurzfristig Arbeit zu finden. Drei Viertel der Befragten fühlen sich in ErRhamna nicht isoliert und rund 40 Prozent der Beschäftigten sind nicht auf motorisierten Transport angewiesen, um zur Arbeit zu gelangen. Hieraus speist sich auch Skepsis gegenüber Umsiedlungen: „Ich würde gerne in einer richtigen Wohnung wohnen, aber es würde weit weg sein und wir wären isoliert und hätten nichts zu tun.“ (23-jähriger arbeitsloser IT-Techniker)

\subsection{Agency und soziale Mobilität in Er-Rhamna}

Zaki (2005: 65 f.) betont, dass sich aus dem Bild einer Siedlung voller Armer in den Augen der Öffentlichkeit eine in gewissem Maße widerwillig akzeptierte Notwendigkeit staatlicher Hilfen ableitet. Auf politischer und gesellschaftlicher Ebene wird den Bewohner*innen damit einhergehend jegliche agency abgesprochen, sich selbst helfen zu können (vgl. Holston/Caldeira 2008; Husseini de Araújo/Batista da Costa 2017: 160). Im Umkehrschluss ergibt sich daraus die Annahme, dass niemand im bidonville wohnt beziehungsweise wohnen bleibt, der über die Ressourcen verfügt, wegziehen zu können. Daraus leitet sich ein weit verbreitetes Misstrauen insbesondere gegenüber jenen Bidonville-Bewohner*innen ab, die ausreichend Geld zur Verfügung haben, um mehr als nur ihre Grundbedürfnisse zu decken, oder die relativ neu zugezogen sind. Ihnen wird unterstellt zu schmarotzen und staatliche Hilfen für den Eigentumserwerb erschleichen zu wollen. So berichtete zum Beispiel eine 22-jährige Studentin einer Privatuniversität, dass sie es zu vermeiden versucht, zu sagen, wo sie wohnt: „Wenn mich in der Schule Freund*innen fragen, wo ich wohne und ich antworte ,im bidonville, dann gucken sie mich komisch an und fragen: ,Und dein Vater kann sich das leisten, für deine Uni zu bezahlen?` Das ist einfach nervig!“ Ihr Vater hat über Jahre gespart, um die monatlichen Studiengebühren beider Töchter in Höhe von drei Vierteln seines Monatsgehalts als Bauarbeiter bezahlen zu können.

Stigmatisierung untergräbt somit die agency der Bewohner*innen, selbst zu entscheiden, wo sie wohnen wollen. Wie in anderen sozial benachteiligten Vierteln auch, wohnen längst nicht alle Bewohner*innen nur in ErRhamna, weil sie es sich nicht leisten können wegzuziehen. Zwar steht die Mehrheit der Bewohner*innen einer möglichen Umsiedlung positiv gegenüber, knapp 65 Prozent der Befragten gaben aber an, ihr Viertel zu mögen/ lieben (fr.: aimer). Einige spüren eine starke Bindung zum Viertel und zum Stadtteil, wie beispielsweise eine vierfache Mutter in Er-Rhamna: „Ich liebe Sidi Moumen! Ich bin hier aufgewachsen und kenne hier jede Straße!“ Für andere ist auch das selbstgebaute Haus ein Bezugspunkt, an den starke Emotionen geknüpft sind: „Ich möchte nicht ausziehen! Ich bin stolz auf mein Haus! Mein Mann und ich haben es mit unseren eigenen Händen gebaut.“ (54-jährige Mutter) Für andere wiederum ist das Zusammenleben mit der Familie ein wesentlicher Faktor des place attachments. Der Fall einer 27-jährigen, alleinwohnenden Unternehmerin sticht hier heraus. Sie hat von ihrem Haus in Er-Rhamna aus selbstständig einen erfolgreichen Internetversandhandel für Kosmetika aufgebaut und besucht alle zwei Jahre eine internationale Messe in Malaysia. Sie gab an, zwar prinzipiell ausziehen zu wollen, aber einfach nicht die Zeit zu finden, nach einer Wohnung zu 
suchen. Außerdem würde sie die Nähe zu ihrer Familie genießen, die gegenüber wohnt. Dieses Beispiel dokumentiert zudem - in deutlichem Kontrast zum Stigma - die existierende, zum Teil beeindruckende agency der Bewohner*innen in Er-Rhamna. Um überhaupt ihr Unternehmen aufbauen zu können, musste die Unternehmerin beispielsweise das Internetkabel eines einige Blöcke entfernten Internetcafés zu ihrem Haus verlängern. Von staatlicher Seite ist es Telekommunikationsunternehmen untersagt, in bidonvilles Festnetzanschlüsse zu installieren und somit nicht-mobilen Internetzugang zu ermöglichen. Die agency der Bewohner*innen wird also bewusst nicht gefördert.

Das Beispiel soll aber nicht dazu dienen, ein international seit den 200oerJahren populäres Image von Slums als Orten des heroischen Unternehmertums zu befeuern and das Konzept der agency auf unternehmerische Aktivität zu reduzieren (vgl. Roy 2011). Im Gegenteil soll es die Heterogenität der Lebens- und Arbeitsweisen in bidonvilles unterstreichen. Etwa die Hälfte der Beschäftigten in Er-Rhamna ist selbstständig, was etwa Tagelöhner*innen, selbstständige Handwerker*innen, Straßenverkäufer*innen und Markthändler*innen, aber auch Kleinunternehmer*innen mit einschließt, die selbst Mitarbeiter*innen beschäftigen. Die andere Hälfte sind Arbeiter*innen und Angestellte, die vor allem in den nahen Industrien Arbeit finden, aber auch im Einzelhandel, der Sicherheits- und Transportbranche und der öffentlichen Verwaltung beschäftigt sind. Knapp zehn Prozent aller Beschäftigten sind Büro- und Fachangestellte beziehungsweise Lehrer*innen.

Die für soziale Mobilität bedeutsame Integration in den Arbeitsmarkt und die agency der Berufswahl werden allerdings durch staatlich (re-)produzierte Stigmatisierung erschwert. Insbesondere männlichen Slumbewohnern hängt das Stigma von ,Ganoven' und ,Taugenichtsen' an, denen man nicht vertrauen kann (Zaki 2005: 62 f.). Zahlreiche Befragte beklagten Diskriminierung auf dem Arbeitsmarkt. „Wenn du sagst, du wohnst im bidonville, dann sagen die Arbeitgeber*innen nur: ,Ich rufe zurück', und du hörst nie wieder was von ihnen“, schilderte zum Beispiel Nachhilfelehrer Khalid. Ein 29-jähriger arbeitsloser Kesselschmied hat, obwohl er gerne in Er-Rhamna lebt, auf seinem Pass die Adresse seiner Tante im benachbarten Aïn Sebaâ eintragen lassen, um auf dem Arbeitsmarkt bessere Chancen zu haben. Diese Beispiele zeigen, dass staatlich (re-)produzierte Stigmata die Entwicklung und die soziale Mobilität der Bewohner*innen hemmen.

Von Seiten der Haushalte hingegen wird aktiv in Aus- und Fortbildung investiert. Im Durchschnitt geben die Haushalte, exklusive Transport, monatlich 158 Dirham (ca. 15 Euro) pro Kopf für die Ausbildung der Kinder aus. Dies ist ein moderat hoher Wert angesichts der Tatsache, dass öffentliche Bildung (Schule und Uni) in Marokko kostenfrei ist und Gebühren somit nur für Bücher, Privatschulen und Nachhilfe anfallen. Ein Viertel der 16- bis 25-Jährigen besucht zudem eine Universität oder ein Zentrum für berufliche Ausbildung. Für viele Familien sind es explizit die Bedingungen im bidonville, die erhöhte Investitionen in die Bildung der Kinder ermöglichen. Dies gilt zum einen, weil Kosten für die Miete gespart werden, zum anderen, weil die Lage Zugang zu den Bildungsinstitutionen ermöglicht. In einem Fall schilderte eine Schülerin, dass ihr Vater sie zu Verwandten nach Er-Rhamna geschickt habe, damit sie ihre Schulbildung fortsetzen könne, da sie auf dem 
Land hierzu keine Möglichkeit gehabt hätte. Spezifische Bedingungen in Slums können somit soziale Mobilität fördern.

\subsection{Land-Stadt-Migration und urban citizenship in Er-Rhamna}

Besonders weit verbreitet ist die diskriminierende Auffassung, dass Bidonville-Bewohner*innen Migrant*innen vom Land sind, die sich ihre vormodernen ländlichen Lebensweisen bewahrt hätten und nicht ausreichend gebildet seien, um zu wissen, wie man sich in der Stadt benimmt (Arrif 1999: 313; Zaki 2005: 67 ff.). Die legitime Zugehörigkeit zur Stadt wird strikt abgesprochen und selbst Vergleiche mit Tieren sind nicht selten (Bénit-Gbaffou/ Oldfield 2014). Die Bidonville-Bewohner*innen repräsentieren im öffentlichen Diskurs all das, was ,legitime“ Stadtbewohner*innen nicht sind oder nicht sein wollen (Zaki 2005: 62). Nahezu alle Bewohner*innen in ErRhamna berichteten von Erfahrungen, bei denen ihnen aktiv urban citizenship abgesprochen wurde. So schilderte zum Beispiel ein Bewohner, dass die Stadtteilverwaltung auf seiner Geburtsurkunde als Wohnort ,koukh' (eine rudimentäre ländliche Hütte) eingetragen hätte, obwohl er in Er-Rhamna geboren war.

Dabei sind bidonvilles spätestens seit Ende der 1980er Jahre nicht (mehr) das primäre Habitat für Neuankömmlinge vom Land (Zaki 2005: 71; Rachik 2012: 317). Während die öffentliche Wahrnehmung weiterhin städtische Probleme auf eine Invasion vom Land reduziert, nehmen bidonvilles zunehmend innerstädtisch verdrängte Personengruppen auf(Rachik 2012: 321). Die Daten aus Er-Rhamna zeigen, dass lediglich 15 Prozent der Befragten direkt aus einem ländlichen Raum, oft in der Nähe von Casablanca, nach ErRhamna gezogen sind. In wenigen Fällen betrifft das den gesamten Haushalt; meist handelt es sich um Einzelpersonen, die beispielsweise durch Heirat nach Er-Rhamna gezogen sind. Die meisten Befragten sind in Er-Rhamna geboren und haben nie woanders gelebt (40 Prozent). Zudem ist der Anteil jener bedeutend, die aus Mietwohnungen in anderen Stadtteilen Casablancas nach Er-Rhamna gezogen (36 Prozent) beziehungsweise dorthin „verdrängt“ (Rachik 2012: 317) worden sind (vgl. Abb. 4). In welchem Maße man diese bedeutende intraurbane Migration als Verdrängung bezeichnen kann, ist schwierig zu bewerten. Wenngleich viele als Grund für den Umzug nach Er-Rhamna Probleme mit Mietzahlungen angaben, betrachteten dieselben

Abb. 4 Die letzten ehemaligen Wohnorte der interviewten Personen vor ihrem Umzug nach Er-Rhamna (Quelle: eigene Daten)

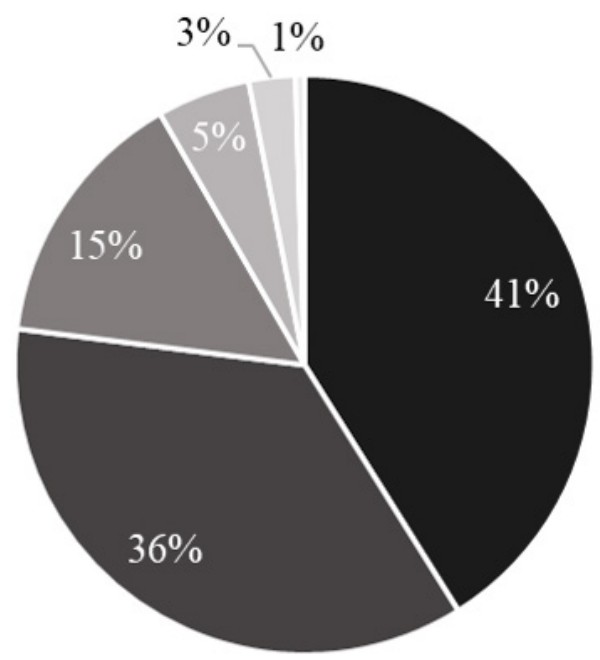

- Mietwohnung in Casablanca

- In Er-Rhamna geboren

- Ländlicher Raum und Umland

- Anderes bidonville in Casablanca

- Andere Stadt in Marokko Ausland 
Personen ihren Umzug nicht zwingend als einen sozialen Abstieg, sondern als Wegfall von ökonomischem Druck und als ein Gewinn von emotionaler und sozialer Sicherheit (fr.: tranquilité) (vgl. Watt 2018). Viele geben an, froh zu sein, immerhin nicht zur Miete wohnen zu müssen, was nahelegt, dass sie den Wohnraum bidonville positiver bewerten als Mietwohnungen. Viele Familien können sich zudem nur ein Zimmer in einer Zweckwohngemeinschaft leisten, was Privatsphäre und Wohnkomfort stark einschränkt und für viele eine fast schon traumatische Erfahrung darstellt.

\section{Wie und warum wir Slums als gewöhnliche Viertel verstehen sollten}

Wie kein zweiter Stadtraum ist der ,Slum' ein Ort der kollektiven Stigmatisierung und der Herabwürdigung, was zu einer sozial konstruierten Überhöhung der Unterschiede zwischen ,formellen' und ,informellen' Vierteln beigetragen hat. Das empirische Beispiel aus Casablanca zeigt, dass das Stigma des Slums für die Bewohner*innen nicht abstrakt ist, sondern in Form von Diskriminierung und infrastrukturellen Benachteiligungen alltäglich spürbar ihr Leben beeinflusst. Zudem hat der Staat durch das gezielte Streuen von Gerüchten und Vorurteilen die Integration der Bewohner*innen und die inkrementelle Entwicklung des Viertels aktiv gebremst und somit selbst Ausgrenzung gefördert. Dabei stehen die einzelnen Bestandteile der kollektiven Stigmatisierung mitunter in starkem Kontrast zu den heterogenen Lebensbedingungen und -weisen in Er-Rhamna, die in der empirischen Forschung zutage getreten sind.

Dementsprechend plädiert dieser Artikel für einen nüchternen, unvoreingenommenen Blick auf die Realitäten in ,Slums', um externen Sichtweisen und gängigen Mythen entgegenzuwirken. Weder sollten Slums als Symbol der vermeintlich unterentwickelten Megastadt, noch als romantisierte Orte heroischen Unternehmer*innentums verstanden werden (vgl. Roy 2011; Perlman 2016; Deboulet 2016). Vielmehr sollten Wissenschaft und Praxis „Slums` als gewöhnliche Viertel (ordinary neighbourhoods) betrachten. Gewöhnliche Viertel sind Viertel mit und ohne Armut, mit prekären und komfortablen Wohnbedingungen, mit Fluktuation, agency und sozialer Mobilität - nicht per se mehr, aber eben auch nicht per se weniger als in anderen Stadtvierteln. Im Gegensatz zum Image des Slums sind gewöhnliche Viertel mehr oder weniger heterogen und es braucht empirische Forschung, um sie zu verstehen. Nur eine solche Sichtweise kann sich von einer global homogenisierenden, kolonial und modernistisch geprägten Außenperspektive lösen, die sich explizit und implizit hinter globalen Entwicklungsagenden verbirgt. Analog zu Robinsons Konzept der ordinary cities stellt sich ein Verständnis von ordinary neighbourhoods gegen global projizierte Dualismen von megacities und global cities, die sich auf städtischer Ebene in international vermarkteten Megaprojekten auf der einen und dem Wunsch nach ,Städten ohne Slums' auf der anderen Seite widerspiegeln (vgl. Huchzermeyer 2011a; Pieterse 2008: $16 \mathrm{ff}$.).

Was bedeutet es also, Slums als gewöhnliche Viertel zu sehen? Hierfür lassen sich in Anlehnung an Robinson drei Notwendigkeiten unterschieden, und zwar 1) weniger globale Homogenisierung, 2) mehr Verständnis für 
innerstädtische Zusammenhänge und 3) die Anerkennung heterogener Bewohner*innenperspektiven.

Erstens ist es notwendig eine oft implizite Tendenz aufzugeben, ,Slums weltweit unter denselben Annahmen zu homogenisieren und zu bewerten. Analog zur Kritik an der Dominanz weniger Städte des Globalen Nordens bei der internationalen Theoriebildung innerhalb der Stadtforschung (Robinson 2002; Schindler 2017) darf ein von wenigen Stadtkontexten (vor allem Mumbai, Nairobi und Rio de Janeiro) geprägtes konzeptionelles Verständnis von ,Slums“ nicht zu einer Verallgemeinerung verschiedenartiger Lebensbedingungen führen. Das Beispiel von Er-Rhamna zeigt, dass unvorsichtig verallgemeinernde Vergleiche mit anderen, ebenso als ,Slums bezeichneten Vierteln nicht mehr bewirken, als Stigmatisierung weiter zu verstärken. Was hat Er-Rhamna gemeinsam mit Vierteln wie Kibera in Nairobi, wo die Mehrheit der Haushalte informell zur Miete wohnt (Schramm 2017), mit Ezbet-en-Nakhl in Kairo, wo Lebensunterhalte nahezu ausschließlich von Müllentsorgung und -recycling abhängen (Didero/ Schneiders 2013), oder mit Makoko in Lagos, wo viele Häuser auf Pfählen im Wasser errichtet worden sind (Adelekan 2010)? Es sollte vielmehr die Aufgabe einer postkolonialen kritischen Forschung sein, nicht primär die vermeintlichen Gemeinsamkeiten zu betonen, sondern auf vergleichende Weise spezifische Unterschiede von gewöhnlichen Vierteln zu analysieren, auf deren Basis sich kontextuell eingebettete Handlungsempfehlungen ableiten lassen.

Zweitens muss eine Sichtweise betont werden, die auch auf Stadtebene Stadtviertel nicht voreingenommen klassifiziert, sondern Zusammenhänge herstellt. Ähnlich der kontrovers diskutierten Frage nach den Gemeinsamkeiten von Städten des Globalen Südens (Schindler 2017; Lawhon/ Truelove 2020) muss die Frage gestellt werden, ob ,Slums ‘ in jeglicher Form anders sind. Das Beispiel von Er-Rhamna zeigt, dass ,Slums ‘ Eigenschaften mit anderen Stadtvierteln teilen und über den Vergleich mit anderen, $g e-$ wöhnlichen Stadtvierteln besser verstanden werden können. Nicht nur erinnern Grundriss, Architektur, und Funktionen des öffentlichen Raums in Teilen von Er-Rhamna stark an die medina; Bewohner*innen wägten ihren Wohnraum auch gegenüber verschiedenen Vor- und Nachteilen von Mietwohnhäusern ab. Analog zur von ordinary cities abgeleiteten Forderung nach einer Stadtgeographie einer world of cities (Robinson 2016a, Lawhon et al. 2020) sollte innerhalb der Stadt in einer weniger vorklassifizierten world of neighbourhoods geforscht werden. Eine kritische postkoloniale Forschung sollte demnach an Vergleichen zwischen gewöhnlichen Wohnvierteln interessiert sein und somit die Annahme des vermeintlich besonderen ,Slums' hinterfragen und intra-städtische Hierarchien überwinden. Verstärkt sollten dabei auch Viertel in Städten des Globalen Südens in den Blick genommen werden, die bislang weniger Aufmerksamkeit erhielten das gilt insbesondere für prekäre Mietswohnungen (Huchzermeyer 2011b; Mayson/Charlton 2015), aber auch für neu entstehende Siedlungen am Stadtrand (Meth 2020; Gastrow 2020).

Drittens ist es notwendig, die Heterogenität innerhalb vermeintlich homogener Wohnviertel zu betonen. Dem Begriff, Slum' und der damit verbundenen Stigmatisierung und Homogenisierung der Bewohner*innen muss 
die kritische Forschung ein Verständnis von ,natürlicher' Heterogenität in gewöhnlichen Vierteln gegenüberstellen. Statt nach einer spezifischen Wirkung von ,Slums' auf die Bewohner*innen zu fragen (Turok/Borel-Saladin 2018), sollte anerkannt werden, dass verschiedene Realitäten in Vierteln koexistieren (vgl. Owusu/Agyei-Mensah/Lund 2008). Das Beispiel aus ErRhamna betont nicht nur das Neben- und Miteinander von Personen mit verschiedenen sozioökonomischen Hintergründen, Herkünften und Wohnsituationen, sondern zeigt auch, wie Perspektiven innerhalb des Viertels, der Nachbarschaft oder auch des Haushalts sich unterscheiden. Das gilt es insbesondere für Handlungsempfehlungen und Politikberatung zu beachten.

Zusammenfassend entsteht hieraus die Forderung nach einer empirischfundierten, kritisch-postkolonialen Sichtweise, die einen unvoreingenommenen, vergleichend-analytischen Blick auf gewöhnliche Viertel wirft und somit die Heterogenität von Wohnvierteln auf globaler, städtischer und lokaler Ebene betont. Das ist insbesondere von Bedeutung im Zuge von globalen Entwicklungsagenden, global zirkulierenden Stadtidealen und -images sowie einer damit einhergehenden Renaissance von standardisierten und oft kontraproduktiven Wohnungsbau- und Umsiedlungsprogrammen. Es ist demnach umso wichtiger, keine universellen Ansprüche zu formulieren, sondern in der Forschung den Kontext einzubeziehen und vergleichend zu analysieren, welche Charakteristika von gewöhnlichen Vierteln sich inwiefern unterscheiden und wie sie sich auf wen konkret auswirken. Nur ein solches Vorgehen kann Stigmatisierung entgegenwirken und eine kreative, progressive und heterogene Umsetzung des Rechts auf angemessenen Wohnraum ermöglichen.

Dieser Artikel wurde durch den Open-Access-Publikationsfonds der DFG der Ruhr-Universität Bochum gefördert.

\section{Endnoten}

[1] ,Bidonville (wörtlich: Kanisterstadt; gewöhnlich übersetzt als ,Slum`) ist eine in Marokko geläufige französische Bezeichnung für Siedlungen, die durch Bewohner*innen selbst ohne Besitztitel und ursprünglich unter Verwendung von einfachen Behelfsbaumaterialien errichtet wurden (Cattedra 2006).

[2] Eine einfache Zufallsstichprobe ist innerhalb von Er-Rhamna u.a. aufgrund nicht zugänglicher Informationen zur Anzahl der Haushalte nicht möglich. Stattdessen wurde mithilfe von Hausnummern ein systematisches Sampling durchgeführt. So konnte eine statistisch zufällige Auswahl unterschiedlicher Bewohner*innen in allen Blöcken Er-Rhamnas gewährleistet werden. Die große Mehrheit stand der Befragung sehr positiv gegenüber. Kaum ein Interview wurde abgelehnt, weshalb Verzerrungen minimiert werden konnten. Die Feldforschung fand zwischen Dezember 2016 und April 2017 in Casablanca statt.

[3] Darija bezeichnet den marokkanischen Dialekt des Arabischen.

[4] Außerhalb des arabischen Sprachraums, wie auch in diesem Artikel, wird der Begriff ,medina benutzt, um die Altstadt einer arabischen Stadt zu beschreiben. Im Arabischen bedeutet ,medina jedoch nur ,Stadt', weswegen in Marokko für ,Altstadt' spezifischere Begriffe gebräuchlich sind, wie ancienne médina (fr.) oder medina al-qadima (ar.).

[5] Alle Vornamen in diesem Artikel sind Pseudonyme.

[6] Alle Zitate der interviewten Bewohner*innen wurden durch Übersetzer*innen ursprünglich von Darija ins Französische und nun durch den Autor weiter ins Deutsche übersetzt. 
[7] Für eine kritische Auseinandersetzung mit dem kolonial geprägten Begriff der ,orientalischen Stadt‘ empfiehlt sich El-Kazaz/Mazur (2017).

[8] Die Auflösung des Rätsels aus Abbildung 1: A: Er-Rhamna, März 2017; B: Medina Rabat, März 2015; C: Er-Rhamna, März 2017; D: Er-Rhamna, Dezember 2016; E: Medina Rabat, März 2015.

[9] Eine 17 Jahre alte Bewohnerin berichtete im Dezember 2016, dass es seit ihrer Geburt jeden Monat heiße, nächsten Monat werde es mit der Umsiedlung losgehen.

[10] ,Karyan' ist in Marokko ein anderer Begriff für ,bidonville oder ,Slum‘

[11] Der qaïd ist ein bestellter Vertreter des Innenministeriums auf der zweitniedrigsten Ebene, dem arrondissement (Stadtteil). Der mqadim ist dem qaïd unterstellt und operiert auf der Blockebene.

[12] Die Geschichte der Stromversorgung in Casablancas Slums ist zu komplex, um hier genauer beschrieben werden zu können. Für eine ausführliche Diskussion siehe NavezBouchanine (2012) und Zaki (2010).

\section{Autor_innen}

Raffael Beier ist Stadtgeograph und forscht insbesondere zu Umsiedlungen und Wohnungsbauprojekten im Nahen Osten sowie in Nord- und Sub-Sahara-Afrika.

raffael.beier@rub.de

\section{Literatur}

Adelekan, Ibidun O. (2010): Vulnerability of poor urban coastal communities to flooding in Lagos, Nigeria. In: Environment and Urbanization 22/2, 433-450.

Arabindoo, Pushpa (2011): Rhetoric of the ,Slum'. Rethinking Urban Poverty. In: City 15/6, 636-646.

Arandel, Christian / Wetterberg, Anna (2013): Between „Authoritarian“ and „Empowered“ Slum Relocation. Social Mediation in the Case of Ennakhil, Morocco. In: Cities 30, 140-148.

Arrif, Abdelmajid (1999): Les compétences citadins à l'épreuve de l'exclusion. L'exemple du bidonvilles de Ben M'Sik à Casablanca (Maroc). In: Pierre Signoles / Galila El Kadi / Rachid Sidi Boumedine (Hg.), L'Urbain dans le monde arabe. Politiques, instruments et acteurs. Paris: CNRS Éditions, 297-317.

Bartoli, Sarah (2011): „Eliminer les bidonvilles = éliminer la pauvreté“, ou les charmes pervers d'une fausse évidence. In: L'Economie politique 49, 44-6o.

Bayat, Asef / Denis, Eric (2000): Who is Afraid of Ashwaiyyat? Urban Change and Politics in Egypt. In: Environment and Urbanization 12/2, 185-199.

Beier, Raffael (2018): Towards a New Perspective on the Role of the City in Social Movements. Urban Policy after the ,Arab Spring'. In: City 22/2, 220-235.

Beier, Raffael (2019): From the City to the Desert. Analysing Shantytown Resettlement in Casablanca, Morocco, from Residents' Perspectives. Berlin: Logos.

Beier, Raffael / Vilmondes Alves, Mariana A. (2015): Die Dominanz des Quantitativen. Das Dilemma des Rechts auf angemessenen Wohnraum in Brasilien, Marokko und Südafrika. In: TRIALOG 121/122, 40-45.

Bénit-Gbaffou, Claire / Oldfield, Sophie (2014): Claiming ,Rights' in the African City. Popular Mobilization and the Politics of Informality. In: Sue Parnell / Sophie Oldfield (Hg.), The Routledge Handbook on Cities of the Global South. Abingdon/New York: Routledge, 281-295.

Benlahcen Tlemçani, Mohamed / Missamou, Rufin (2000): Habitat clandestin et insalubre au Maroc. Vers une stratégie d'intervention plurielle. In: Les Annales de la Recherche Urbaine 86, 111-118.

Berner, Erhard (2016): „Zur Wohnungsfrage“ im 21. Jahrhundert. Marktversagen, hilflose Politik und die globale Ausbreitung von Slums. In: Peripherie 36/141, 10-31.

Berry-Chikhaoui, Isabelle (2010): Major Urban Projects and the People Affected. The Case of Casablanca's Avenue Royale. In: Built Environment 36/2, 216-229. 
Bogaert, Koenraad (2011): The Problem of Slums. Shifting Methods of Neoliberal Urban Government in Morocco. In: Development and Change 42/3, 709-731.

Bogaert, Koenraad (2018): Globalized Authoritarianism. Megaprojects, Slums, and Class Relations in Urban Morocco. Minneapolis: University of Minnesota Press.

Buckley, Robert M. / Kallergis, Achilles / Wainer, Laura (2016): Addressing the Housing Challenge. Avoiding the Ozymandias Syndrome. In: Environment and Urbanization 28/1, 119-138.

Bundesministerium für wirtschaftliche Zusammenarbeit und Entwicklung (BMZ) (о. J.): MDG 7. Die ökologische Nachhaltigkeit sichern. http://www.bmz.de/de/themen/2030_ agenda/historie/MDGs_2015/fortschritte/mdg7/index.html (letzter Zugriff am 22.4.2020).

Cattedra, Raffaele (2006): Bidonville. Paradigme et réalité refoulée de la ville du XXe siècle. In: Jean-Charles Depaule (Hg.), Les mots de la stigmatisation urbaine. Paris: Éditions UNESCO; Éditions de la Maison des sciences de l'homme, 123-163.

Cavalcanti, Mariana (2014): Threshold Markets. The Production of Real-Estate Value between the ,Favela' and the ,Pavement'. In: Brodwyn Fischer / Bryan McCann / Javier Auyero (Hg.), Cities From Scratch. Poverty and Informality in Urban Latin America. Durham: Duke University Press, 208-237.

Croese, Sylvia / Cirolia, Liza Rose / Graham, Nick (2016): Towards Habitat III. Confronting the Disjuncture between Global Policy and Local Practice on Africa's ,Challenge of Slums'. In: Habitat International 53, 237-242.

Davis, Mike (2007): Planet der Slums. Berlin: Assoziation A.

Deboulet, Agnès (2016): Rethinking Precarious Neighborhoods. Knowledge and Recognition. In: Agnès Deboulet (Hg.), Rethinking Precarious Neighbourhoods. Paris: Agence Française de Développement, 9-35.

Didero, Maike / Schneiders, Thorsten Gerald (2013): Zabbalin - Menschen im Müll. Untersuchungen zur Geschichte, Gegenwart und Zukunft der informellen Abfallwirtschaft in Kairo. In: Thorsten Gerald Schneiders (Hg.), Die Araber im 21. Jahrhundert. Politik, Gesellschaft, Kultur. Wiesbaden: Springer, 133-159.

El-Kazaz, Sarah / Mazur, Kevin (2017): Introduction to Special Section. The Un-Exceptional Middle Eastern City. In: City \& Society 29/1, 148-161.

Escher, Anton / Petermann, Sandra / Arnold, Gregor (2018): Das neue Marrakech. Eine Materialisierung des Mythos von Tausendundeiner Nacht? In: Geographische Rundschau $7 / 8,52-57$.

Fischer, Brodwyn (2014): A Century in the Present Tense. Crisis, Politics, and the Intellectual History of Brazil's Informal Cities. In: Brodwyn Fischer / Bryan McCann / Javier Auyero (Hg.), Cities From Scratch. Poverty and Informality in Urban Latin America. Durham: Duke University Press, 9-67.

Fleischer, Friederike / Hurtado Tarazona, Adriana / Alvarez Rivadulla, Maria Jose (2019): Ghettos am Rande der Stadt? Sozialwohnungsbaupolitik und soziale Ungleichheit in Kolumbien. In: sub \urban. zeitschrift für kritische stadtforschung 7/3, 27-46.

Friedmann, John (1986): The World City Hypothesis. In: Development and Change 17/1, 69-83.

Gastrow, Claudia (2020): Housing Middle-classness. Formality and the Making of Distinction in Luanda. In: Africa 90/3, 509-528.

Geiselhart, Klaus (2017): Call it by its proper name! Territory-ism and territorial stigmatisation as a dynamic model: the case of Old Naledi. In: Paul Kirkness / Andreas Tijé-Dra (Hg.), Negative neighbourhood reputation and place attachment. The production and contestation of territorial stigma. Abingdon/New York: Routledge, 216-234.

Ghertner, D. Asher (2012): Nuisance Talk and the Propriety of Property. Middle Class Discourses of a Slum-Free Delhi. In: Antipode 44/4, 1161-1187.

Gilbert, Alan (2007): The Return of the Slum: Does Language Matter? In: International Journal of Urban and Regional Research 31/4, 697-713.

Haut-Commisariat au Plan (HCP) (2018): Niveau de vie et pauvreté. https://www.hcp.ma/ downloads/Niveau-de-vie-et-pauvrete_t11884.html (letzter Zugriff am 16.4.2020).

Holston, James / Caldeira, Teresa P. R. (2008): Urban Peripheries and the Invention of Citizenship. In: Harvard Design Magazine 28, 18-23.

Huchzermeyer, Marie (2011a): Cities With ,Slums‘. From Informal Settlement Eradication to a Right to the City in Africa. Claremont: UCT Press.

Huchzermeyer, Marie (2011b): Tenement Cities. From 19th Century Berlin to 21st Century Nairobi. Trenton: Africa World Press. 
Huchzermeyer, Marie (2013): ,Slum‘ Upgrading or ,Slum‘ Eradication? The Mixed Message of the MDGs. In: Andrew Sumner / Alicia Ely Yamin / Malcolm Langford (Hg.), The Millennium Development Goals and Human Rights. Past, Present and Future. Cambridge: Cambridge University Press, 295-315.

Husseini de Araújo, Shadia / Batista da Costa, Everaldo (2017): From Social Hell to Heaven? The intermingling processes of territorial stigmatisation, agency from below and gentrification in the Varjão, Brazil. In: Paul Kirkness / Andreas Tijé-Dra (Hg.), Negative neighbourhood reputation and place attachment. The production and contestation of territorial stigma. Abingdon/New York: Routledge, 158-177.

Lawhon, Mary / Le Roux, Lené / Makina, Anesu / Nsangi, Gloria / Singh, Aditi / Sseviiri, Hakimu (2020): Beyond southern urbanism? Imagining an urban geography of a world of cities. In: Urban Geography 72/2, 1-11.

Lawhon, Mary / Truelove, Yaffa (2020): Disambiguating the southern urban critique. Propositions, pathways and possibilities for a more global urban studies. In: Urban Studies 57/1, 3-20.

Mayson, Simon Sizwe / Charlton, Sarah (2015): Accommodation and Tenuous Livelihoods in Johannesburg's Inner City. The ,Rooms` and ,Spaces' Typologies. In: Urban Forum 26/3, 343-372.

McFarlane, Colin (2008): Urban Shadows. Materiality, the ,Southern City and Urban Theory. In: Geography Compass 2/2, 340-358.

Meth, Paula (2020): ,Marginalised formalisation‘. An analysis of the in/formal binary through shifting policy and everyday experiences of ,poor ${ }^{6}$ housing in South Africa. In: International Development Planning Review 42/2, 139-164.

Ministère del'Habitat, de l'Urbanisme et de la Politique de la Ville (MHUPV) (2012): Rapport national - Résorption des bidonvilles: l'expérience marocaine. Conférence internationale „Sortir des bidonvilles: un défi mondial pour 2020“, Novembre 2012. Rabat.

Ministère del'Habitat, del'Urbanisme et de la Politique de la Ville(MHUPV) (2013): Discours et Interviews de Sa Majesté le Roi Mohammed VI. „Habitat, Urbanisme et Politique de la Ville“. https://aua.ma/wp-content/uploads/2018/02/Royaume-du-Maroc.pdf (letzter Zugriff am 11.4.2020).

Navez-Bouchanine, Françoise (2012): Les nouvelles voies de la négociation dans les politiques de résorption des bidonvilles au Maroc. Entre recasement et accompagnement social. In: Françoise Navez-Bouchanine / Agnès Deboulet (Hg.), Effets sociaux des politiques urbaines. L'entre-deux des politiques institutionnelles et des dynamiques sociales: Algérie, Maroc, Liban, Mauritanie. Paris u. a.: Éditions Karthala u.a., 166-218.

Nikuze, Alice / Sliuzas, Richard / Flacke, Johannes / van Maarseveen, Martin (2019): Livelihood Impacts of Displacement and Resettlement on Informal Households. A Case Study from Kigali, Rwanda. In: Habitat International 86, 38-47.

Owusu, George / Agyei-Mensah, Samuel / Lund, Ragnhild (2008): Slums of Hope and Slums of Despair. Mobility and Livelihoods in Nima, Accra. In: Norwegian Journal of Geography 62/3, 180-190.

Patel, Sejal / Sliuzas, Richard / Mathur, Navdeep (2015): The Risk of Impoverishment in Urban Development-Induced Displacement and Resettlement in Ahmedabad. In: Environment and Urbanization 27/1, 231-256.

Perlman, Janice (2016): Rethinking precarious neighborhoods. Concepts and consequences of marginality. In: Agnès Deboulet (Hg.), Rethinking Precarious Neighborhoods. Paris: Agence Française de Développement, 39-56.

Pieterse, Edgar A. (2008): City futures. Confronting the crisis of urban development. Kapstadt u. a.: UCT Press, Zed Books.

Rachik, Abderrahmane (2012): Casablanca: Ein ländliches Zuwanderungsgebiet? In: Jörg Gertel / Ingo Breuer (Hg.), Alltagsmobilitäten. Aufbruch marokkanischer Lebenswelten. Bielefeld: transcript, 317-326.

Robinson, Jennifer (2002): Global and World Cities. A View from off the Map. In: International Journal of Urban and Regional Research 26/3, 531-554.

Robinson, Jennifer (2016a): Comparative Urbanism. New Geographies and Cultures of Theorizing the Urban. In: International Journal of Urban and Regional Research 40/1, 187-199.

Robinson, Jennifer (2016b): Thinking cities through elsewhere. Comparative tactics for a more global urban studies. In: Progress in Human Geography 40/1, 3-29.

Robinson, Jennifer (2006): Ordinary cities. Between modernity and development. Abingdon/New York: Routledge. 
Roy, Ananya (2011): Slumdog Cities. Rethinking Subaltern Urbanism. In: International Journal of Urban and Regional Research 35/2, 223-238.

Roy, Ananya (2014): Slum-free cities of the Asian century. Postcolonial Government and the Project of Inclusive Growth. In: Singapore Journal of Tropical Geography 35/1, 136-150.

Sassen, Saskia (2001): The Global City. New York, London, Tokyo. Princeton/Oxford: Princeton University Press.

Schindler, Seth (2017): Towards a paradigm of Southern urbanism. In: City 21/1, 47-64.

Schramm, Sophie (2017): People's Room for Manoeuvre in a Fragmented City. State Housing in Kibera, Nairobi. In: Transformation: Critical Perspectives on Southern Africa 93, 116-141.

Spotorno, Mauro (2019): Gentrification and European Entrepreneurship in Marrakesh. In: Maria Paradiso (Hg.), Mediterranean Mobilities. Europess Changing Relationships. Cham: Springer International Publishing, 167-176.

Statistisches Bundesamt (2020): Indikatoren der UN-Nachhaltigkeitsziele. Ziel 11. https:// sustainabledevelopment-germany.github.io/11/ (letzter Zugriff am 9.4.2020).

Steinbrink, Malte / Ehebrecht, Daniel / Haferburg, Christoph / Deffner, Veronika (2015): Megaevents und favelas. Strategische Intervention und sozialräumliche Effekte in Rio de Janeiro. In: sub \urban. zeitschrift für kritische stadtforschung 3/1, 45-74.

Steinbrink, Malte / Haferburg, Christoph / Ley, Astrid (2011): Festivalisation and urban renewal in the Global South. Socio-spatial consequences of the 2010 FIFA World Cup. In: South African Geographical Journal 93/1, 15-28.

Turner, John F. C. (1977): Housing by People. Towards Autonomy in Building Environments. New York: Pantheon Books.

Turok, Ivan (2016): Housing and the Urban Premium. In: Habitat International 54/3, 234-240.

Turok, Ivan / Borel-Saladin, Jackie (2018): The Theory and Reality of Urban Slums. Pathways-out-of-poverty or Cul-de-sacs? In: Urban Studies 55/4, 767-789.

United Nations Human Settlements Programme (UN-Habitat) (2003): The Challenge of Slums. Global Report on Human Settlements 2003. London/Sterling: Earthscan.

Valladares, Licia do Prado (2019): The Invention of the Favela. Chapel Hill: University of North Carolina Press.

Watson, Vanessa (2009): Seeing from the South. Refocusing Urban Planning on the Globe's Central Urban Issues. In: Urban Studies 46/11, 2259-2275.

Watt, Paul (2018): „This Pain of Moving, Moving, Moving“. Evictions, Displacement, and the Logics of Expulsions in London. In: L'Année Sociologique 68/1, 67-100.

World Bank / United Nations Human Settlements Programme (UN-Habitat) (2000): Cities Alliance for Cities Whithout Slums. Action Pan for Moving Slum Upgrading to Scale. http://www.citiesalliance.org/sites/citiesalliance.org/files/ActionPlan.pdf (letzter Zugriff am 22.1.2014).

Yelling, James Alfred (1986): Slums and Slum Clearance in Victorian London. London: Allen and Unwin.

Yiftachel, Oren (2009): Theoretical Notes on ,Gray Cities'. The Coming of Urban Apartheid? In: Planning Theory 8/1, 88-100.

Zaki, Lamia (2005): Pratiques politiques au bidonville, Casablanca (2000-2005). Paris: Dissertation am Institut d'Etudes Politiques de Paris.

Zaki, Lamia (2010): L'électrification temporaire des bidonvilles casablancais. Aspects et limites d'une transformation. In: Politique africaine 120, 45-66.

Zemni, Sami / Bogaert, Koenraad (2011): Urban Renewal and Social Development in Morocco in an Age of Neoliberal Government. In: Review of African Political Economy 38/129, 403-417.

\section{Ordinary neighbourhoods. Stigma and Reality in Casablanca's Slums}

Globally, slums have become the symbol of underdeveloped and fast-growing megacities of the Global South. The related stigmatisation of slums affects its inhabitants on a daily basis. Latest through the Millennium 
Development Goals, it has further provoked the renaissance of large-scale housing programmes, as well as displacement and resettlement of slum dwellers to urban margins. Focussing on Morocco, the article shows how a global negative image of slums has inspired new, repressive housing policies. The paper builds on an empirical case study from Casablanca, Morocco, including household surveys and qualitative interviews with residents. By deconstructing existing stigmas, the author argues that slums are heterogenous neighbourhoods that do not naturally differ from others. In conclusion, the paper advocates for an understanding of slums as ordinary neighbourhoods - similar to Jennifer Robinson's concept of ordinary cities and, hence, calls for a postcolonial, empirical, and comparative perspective. 
